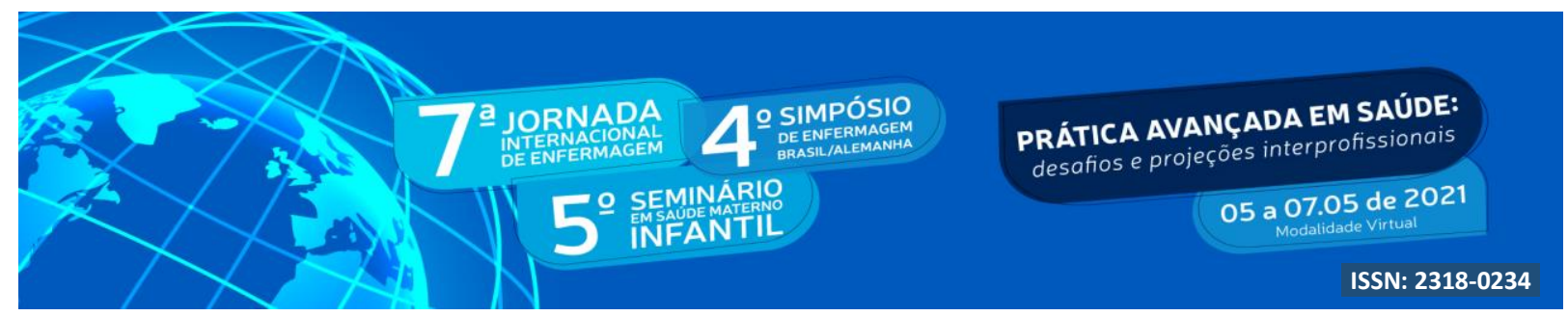

DOI: http://doi.org/10.48195/jie2021-082

\title{
PLANO DE PRECEPTORIA PARA ENFERMEIRAS NEFROLOGISTAS ATUANTES EM HOSPITAL UNIVERSITÁRIO'
}

\author{
Viviane Peixoto dos Santos Pennafort ${ }^{2}$; Ana Elza Oliveira de Mendonça ${ }^{3}$; Eliane Santos \\ Cavalcante $^{4}$; Júlia Silva Fonseca dos Anjos ${ }^{5}$; Aldair de Souza Paiva ${ }^{6}$
}

\begin{abstract}
RESUMO
Objetivou-se desenvolver um plano de preceptoria para enfermeiros do setor de hemodiálise de um hospital universitário.Trata-se de um projeto de intervenção com elaboração de um plano de preceptoria para enfermeiros, por meio da aprendizagem problematizadora fundamentada nas metodologias ativas. O plano foi idealizado para o setor de diálise de um hospital universitário, por meio da matriz SWOT, no período de janeiro a setembro de 2020. Essa estratégia pedagógica priorizou as principais atividades de preceptoria das enfermeiras nefrologistas no acompanhamento do estágio dos discentes dos cursos de graduação, residência e pós-graduação em Enfermagem, como a utilização das metodologias ativas, melhoria da comunicação preceptor-discente-tutor, avaliação contínua do discente e coprodução científica, a partir das vivências no setor. Dessa forma, vislumbrase o aprimoramento das competências técnico-científicas das preceptoras no desempenho de suas atividades como educadoras, capaz de despertar um novo modo de ensinar, sensível às demandas dos discentes.
\end{abstract}

Palavras-chave: Capacitação; Educação em saúde; Enfermagem; Preceptoria.

\begin{abstract}
The objective was to develop a preceptorship plan for nurses in the hemodialysis sector of a university hospital. This is an intervention project with the development of a preceptorship plan for nurses, through problematizing learning based on active methodologies. The plan was devised for the dialysis sector of a university hospital, through the SWOT matrix, from January to September 2020. This pedagogical strategy prioritized the main preceptory activities of nephrologist nurses in monitoring the internship of students of the nursing courses. undergraduate, residency and postgraduate studies in Nursing, such as the use of active methodologies, improvement of teacher-student-tutor communication, continuous student evaluation and scientific co-production, based on experiences in $n$

\footnotetext{
${ }^{1}$ Trabalho de Conclusão de Curso de Especialização em Preceptoria em Saúde da EBSERH.

${ }^{2}$ Doutora em Enfermagem. Docente do Mestrado Profissional em Gestão da Qualidade em Serviços de Saúde. Universidade Federal do Rio Grande do Norte. E-mail: vivipspf@yahoo.com.br

${ }^{3}$ Doutora em Enfermagem. Docente do Mestrado Profissional em Gestão da Qualidade em Serviços de Saúde. Universidade Federal do Rio Grande do Norte. E-mail: anaelza@gmail.com

${ }^{4}$ Doutora em Enfermagem. Docente do Mestrado Profissional em Gestão da Qualidade em Serviços de Saúde. Universidade Federal do Rio Grande do Norte. E-mail: elianeufrn@ hotmail.com

${ }^{5}$ Graduanda em Enfermagem. Universidade Federal do Rio Grande do Norte. E-mail: julia.sanjos18@gmail.com ${ }^{6}$ Doutor em Ciências da Saúde. Universidade Federal do Rio Grande do Norte. E-mail: aldairpaiva100@gmail.com
} 


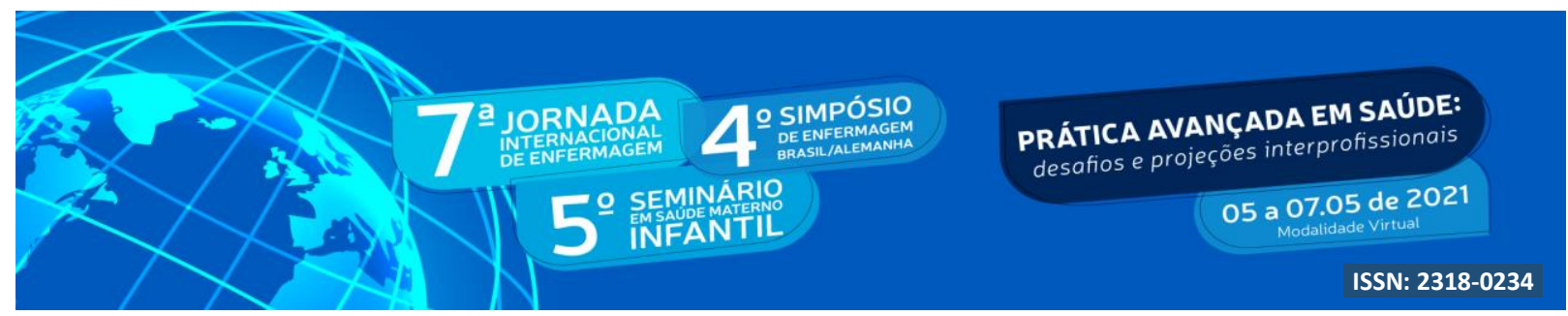

this way, the improvement of the technical-scientific competences of the preceptors is envisaged in the performance of their activities as educators, capable of awakening a new way of teaching, sensitive to the demands of the students.

Key Words: Training; Health education; Nursing; Preceptorship.

\section{INTRODUÇÃO}

Historicamente, o processo de formação profissional na área da saúde do Brasil foi desconsiderado no contexto sociopolítico e pedagógico, observando-se incoerências entre a qualificação dos profissionais e as demandas do Sistema Único de Saúde (SUS) na atenção à saúde.

Alguns pesquisadores ressaltam que a formação de profissionais da saúde deve responder, com qualidade e efetividade, às demandas do SUS e proporcionar, ao profissional, uma atuação que tenha como princípio as necessidades sociais e de saúde de usuários e famílias, em consonância com as Diretrizes Curriculares Nacionais. Entretanto, a desarticulação entre as instituições formadoras e os serviços de saúde tem contribuído para acentuar o distanciamento entre a formação e as necessidades do SUS (ANTUNES; DAHER et al, 2017).

Em contrapartida, os principais desafios no exercício da preceptoria consistem no despreparo pedagógico do preceptor para lidar com as demandas gerais do programa, como avaliar, planejar, desenvolver pesquisas, trabalhar com grupos, com metodologias ativas e em desenvolver ações com profissionais e discentes de outros cursos. Outro desafio consiste na inexistência de flexibilidade curricular em relação aos horários e excesso de demanda de atividades nos serviços de saúde, o que influencia na atenção individual direcionada ao discente e consequentemente, nas experiências no período do estágio e na construção do conhecimento de práticas em saúde (LIMA; ROZENDO, 2015; SILVA; MOREIRA, 2019).

Outro estudo ressaltou como desafio na formação profissional em saúde, a sobrecarga de atividades acadêmicas e a escassez de articulação entre teoria e prática no cotidiano da residência em saúde. Os autores identificaram que a maioria dos residentes multiprofissionais pesquisados apresentou sintomas de estresse em nível considerado não saudável. Os sintomas psicológicos que predominaram foram o cansaço excessivo, vontade de fugir de tudo, angústia diária e dúvida quanto a si próprio, o que pode sinalizar sofrimento psíquico e 


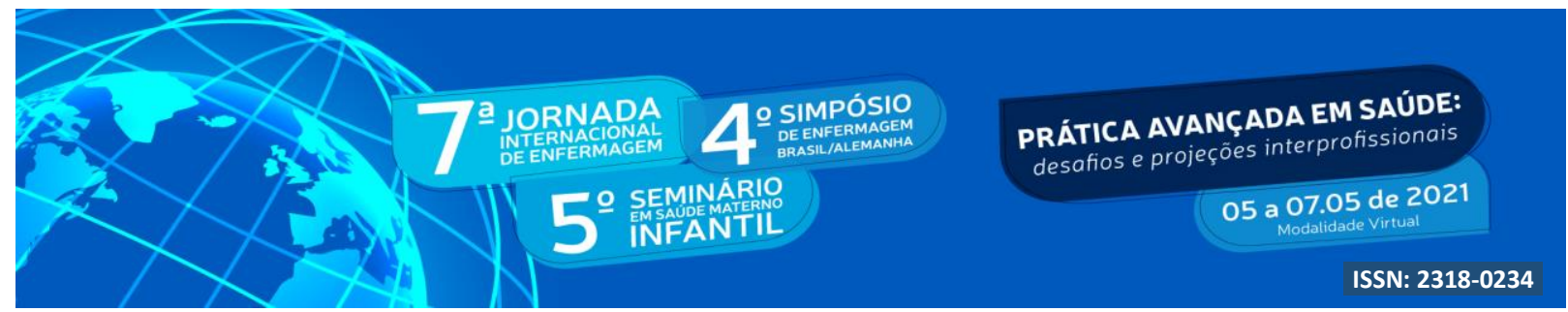

problemas na saúde como um todo. Outrossim, ante à dificuldade de reconhecimento do trabalho por parte da equipe, a preceptoria e a mão de obra barata foram consideradas pelos residentes como fatores relacionados a sentimento de frustração no cotidiano do ensinoserviço (SILVA; MOREIRA, 2019).

Os cursos na área da saúde objetivam instrumentalizar, técnica e cientificamente, os futuros profissionais, complementando o processo de formação. A relação de ensino-aprendizagem ou discente-preceptor, que se efetiva nessa formação prática, deveria se configurar como processo de aprendizagem significativa, no qual ao conhecimento, já experienciado pelo aluno em momentos anteriores, se acresçam às novas vivências, potencializando esse processo e, consequentemente, a assistência prestada aos usuários dos serviços de saúde (ANTUNES; DAHER et al, 2017).

Nesta perspectiva, observou-se a necessidade de elaboração de um Plano de Preceptoria, a fim de mitigar os desafios da preceptoria em saúde por meio do estímulo à prática pedagógica participativa, dialógica e de coprodução de conhecimentos e oportunidades na formação de futuros profissionais. Dessa forma, questionou-se quais estratégias facilitarão uma prática pedagógica inovadora do enfermeiro/preceptor, capaz de envolver os discentes e suas inquietações no processo de formação, com vistas à qualidade da assistência em saúde aos pacientes em tratamento hemodialítico, no setor de diálise de um hospital de ensino?

Vislumbra-se a partir desta proposta, promover um processo efetivo de ensino-aprendizagem entre preceptor e discente com possibilidade de construção e socialização do conhecimento em saúde.

\section{OBJETIVO}

Desenvolver um plano de preceptoria para enfermeiros do setor de hemodiálise de um hospital universitário.

\section{METODOLOGIA}

Trata-se de um projeto de intervenção com elaboração de um Plano de Preceptoria, para enfermeiros atuantes no setor de hemodiálise da Unidade de Sistema Urinário do Hospital Universitário Onofre Lopes (HUOL). 


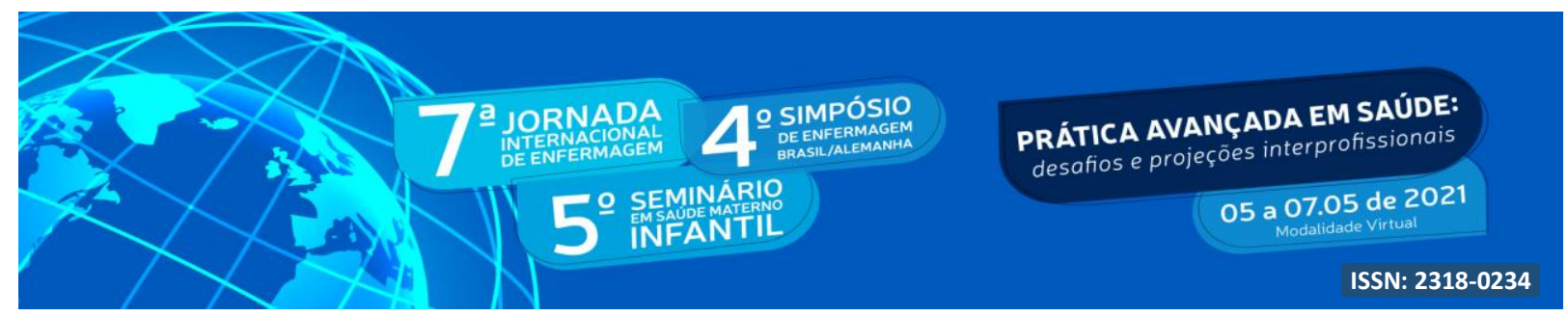

O HUOL faz parte da Universidade Federal do Rio Grande do Norte (UFRN) e atualmente, é administrado pela Empresa Brasileira de Serviços Hospitalares (EBSERH). A instituição é conveniada ao SUS, como referência de média e alta complexidade em diversas áreas para todo o estado do Rio Grande do Norte - RN. O HUOL integra os campos de prática de cursos de graduação e de Pós-Graduação (residência, especialização, mestrado e doutorado) em diversas áreas da saúde da UFRN.

O setor de hemodiálise do HUOL é constituído por cinco leitos e uma sala destinada aos pacientes em isolamento respiratório em tratamento hemodialítico, com atendimento contínuo nos três turnos. A equipe de enfermagem do setor é responsável pelo tratamento hemodialítico dos pacientes das enfermarias, UTI adulta e pediátrica e transplante renal. $\mathrm{O}$ setor recebe graduandos e residentes do curso de Enfermagem e Medicina, além dos estudantes dos cursos de extensão universitária, com permanência média de 15 dias.

A equipe de enfermagem do setor de diálise é formada por nove enfermeiras, destas, oito são especialistas em enfermagem em nefrologia, uma tem doutorado em cuidados clínicos em saúde e outras três, têm mestrado, além de doze técnicos de enfermagem com experiência na área. A equipe é formada ainda, por cinco médicos nefrologistas e atualmente, por três residentes em nefrologia.

Nesta perspectiva, o Plano de Preceptoria foi desenvolvido como estratégia pedagógica para as enfermeiras do setor de hemodiálise, a partir da identificação dos elementos por meio da matriz SWOT, matriz de análise situacional e triângulo de governo, a fim de nortear as atividades de estágio dos Cursos de Graduação, residência e Pós-graduação em Enfermagem.

Dessa forma, a equipe executora será composta por enfermeiras nefrologistas do setor, com participação dos discentes e tutores/docentes envolvidos no processo de ensinoaprendizagem de formação profissional em saúde.

O projeto de intervenção foi norteado pelos procedimentos éticos exigidos pelas Resoluções n510/2016 do Conselho Nacional de Saúde.

\section{RESULTADOS E DISCUSSÃO}




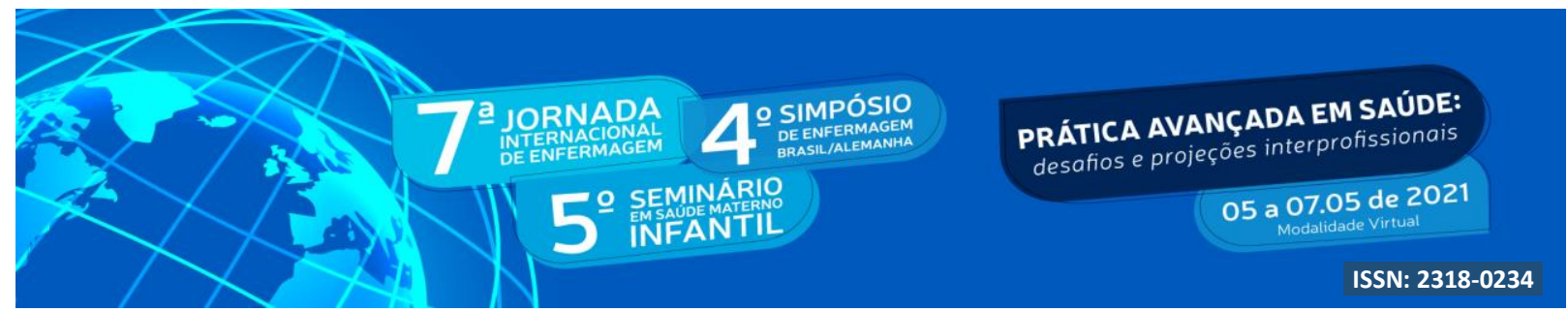

A figura 1 destacou os elementos priorizados no Plano de Preceptoria para enfermeiras nefrologistas do setor de hemodiálise, sendo elencadas as principais ações, a descrição da implementação de cada atividade, assim como, os atores envolvidos e a estrutura mínima necessária para o planejamento do estágio, acolhimento dos discentes e residentes e discussão de casos clínicos durante a realização das atividades. Elementos imprescindíveis para um processo de ensino-aprendizagem criativo, contextualizado e de participação ativa de todos os envolvidos.

Figura 1. Elementos do Plano de Preceptoria para enfermeiros do setor de diálise do HUOL; Natal-

RN, 2020.

\begin{tabular}{|c|c|c|c|}
\hline AÇÕES & DESCRIÇÃO /IMPLEMENTAÇÃO & $\begin{array}{c}\text { ATORES } \\
\text { ENVOLVIDOS }\end{array}$ & $\begin{array}{l}\text { ESTRUTURA } \\
\text { NECESSÁRIA }\end{array}$ \\
\hline $\begin{array}{l}\text { Desenvolver } \\
\text { uma prática } \\
\text { pedagógica } \\
\text { crítica, } \\
\text { dialógica, } \\
\text { interativa e } \\
\text { participativa. } \\
\text { Capacitar os } \\
\text { preceptores para } \\
\text { utilização das } \\
\text { metodologias } \\
\text { ativas. }\end{array}$ & $\begin{array}{l}\text { Utilizar as metodologias ativas de } \\
\text { ensino/aprendizagem como a } \\
\text { Problematização com o Arco de Charles e } \\
\text { Maguerez; Problema Based Learning (PBL) } \\
\text { e simulação realística. } \\
\text { Realizar oficinas com os enfermeiros da } \\
\text { diálise para apresentação das metodologias } \\
\text { ativas, sua finalidade e etapas de } \\
\text { desenvolvimento. } \\
\text { Valorizar a experiência prévia do discente. } \\
\text { Envolver o discente no planejamento das } \\
\text { atividades propostas para o estágio. }\end{array}$ & $\begin{array}{l}\text { Preceptores, } \\
\text { discentes, } \\
\text { docente/tutor. } \\
\text { Docente/tutor } \\
\text { será responsável } \\
\text { pela capacitação } \\
\text { dos enfermeiros } \\
\text { do setor de } \\
\text { diálise. }\end{array}$ & \multirow{4}{*}{$\begin{array}{l}\text { Sala de diálise e } \\
\text { sala de apoio } \\
\text { para atividades } \\
\text { de acolhimento } \\
\text { dos discentes, } \\
\text { orientação de } \\
\text { algumas } \\
\text { atividades, } \\
\text { como } \\
\text { apresentação e } \\
\text { discussão de } \\
\text { casos clínicos e } \\
\text { ainda, para o } \\
\text { planejamento e } \\
\text { avaliação das } \\
\text { atividades de } \\
\text { estágio } \\
\text { capacitação } \\
\text { profissional. }\end{array}$} \\
\hline $\begin{array}{l}\text { Melhorar a } \\
\text { comunicação } \\
\text { com o discente e } \\
\text { docente/tutor }\end{array}$ & $\begin{array}{l}\text { Melhoria da comunicação e atuação } \\
\text { interprofissional entre profissionais, } \\
\text { discentes e docentes/tutores no } \\
\text { planejamento e avaliação das atividades } \\
\text { propostas para o estágio. }\end{array}$ & $\begin{array}{l}\text { Preceptores, } \\
\text { discentes } \\
\text { docente/tutor. }\end{array}$ & \\
\hline $\begin{array}{l}\text { Participação } \\
\text { ativa no } \\
\text { planejamento e } \\
\text { avaliação do } \\
\text { estágio }\end{array}$ & $\begin{array}{l}\text { Avaliar as atividades desenvolvidas a cada } \\
\text { período de estágio. } \\
\text { Solicitar aos gestores em saúde as } \\
\text { mudanças necessárias na infraestrutura do } \\
\text { setor, para acolhimento dos estudantes e } \\
\text { outras atividades inerentes à preceptoria. }\end{array}$ & $\begin{array}{l}\text { Preceptores, } \\
\text { discentes, } \\
\text { docente/tutor. }\end{array}$ & \\
\hline $\begin{array}{l}\text { Estímulo } \\
\text { produçãoo } \\
\text { científica }\end{array}$ & $\begin{array}{l}\text { Desenvolver resumos para congresso e } \\
\text { artigos junto aos discentes, docentes e } \\
\text { demais profissionais, a partir da experiência } \\
\text { no período de estágio. }\end{array}$ & $\begin{array}{l}\text { Preceptores, } \\
\text { discentes, } \\
\text { docente/tutor. }\end{array}$ & \\
\hline
\end{tabular}

Fonte: elaboração própria.

A figura 2 apresenta as oportunidades e as possíveis fragilidades, assim como, os fatores envolvidos por meio da matriz SWOT acerca das atividades de preceptoria realizadas 


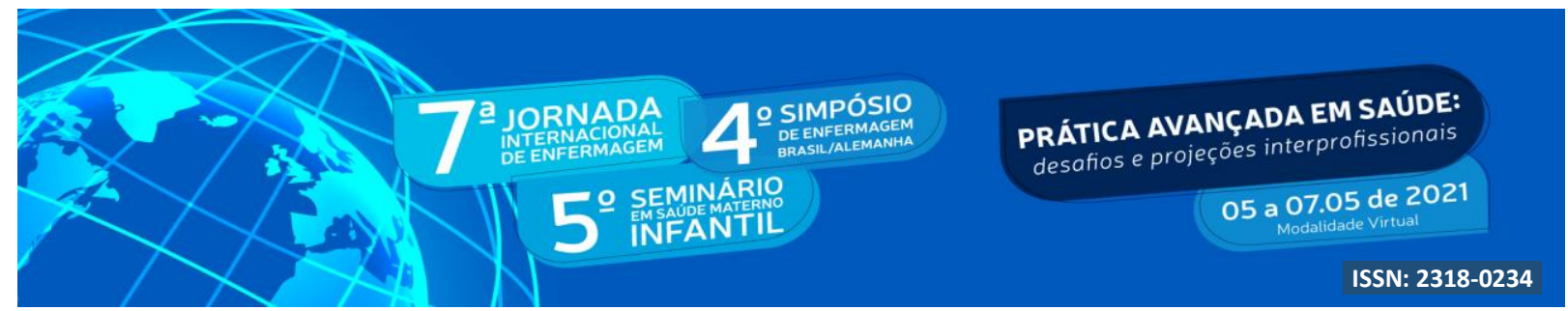

pelas enfermeiras nefrologistas.

Figura 2. Matriz SWOT com descrição das fragilidades e oportunidades na atividade de preceptoria de enfermeiros do setor de diálise do HUOL; Natal-RN, 2020.

\begin{tabular}{|c|c|c|}
\hline \multicolumn{3}{|c|}{$\begin{array}{r}\text { MATRIZ SWOT } \\
\end{array}$} \\
\hline & OPORTUNIDADES & FRAGILIDADES \\
\hline 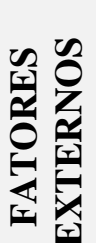 & $\begin{array}{l}\text { - Utilização de metodologias ativas no processo de } \\
\text { ensino } \\
\text { - Desenvolvimento de projetos de extensão e pesquisa } \\
\text { - Participação no planejamento das atividades do } \\
\text { estágio }\end{array}$ & $\begin{array}{l}\text { - Falta de incentivo da gestão } \\
\text { - Infraestrutura inadequada } \\
\text { - Carga-horária insuficiente } \\
\text { - Desvalorização da } \\
\text { preceptoria }\end{array}$ \\
\hline \multirow{3}{*}{ 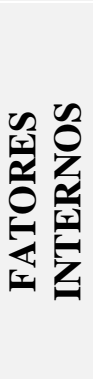 } & FORTES & FRACOS \\
\hline & $\begin{array}{l}\text { - Processo pedagógico de ensino em construção } \\
\text { - Aprimoramento profissional } \\
\text { - Atuação interdisciplinar } \\
\text { - Contribuiçãa com a formação dos profissionais } \\
\text { - Articulação entre as atividades assistenciais e as } \\
\text { atividades de preceptoria }\end{array}$ & $\begin{array}{l}\text { - Despreparo para preceptoria } \\
\text { - Desinteresse dos discentes } \\
\text { - Desarticulação entre teoria e } \\
\text { prática } \\
\text { - Demanda de atividades } \\
\text { assistenciais }\end{array}$ \\
\hline & POSITIVO & NEGATIVO \\
\hline
\end{tabular}

Fonte: elaboração própria.

Considera-se que a implementação do Plano de Preceptoria (PP) para enfermeiros do setor de diálise do Hospital Universitário Onofre Lopes (HUOL) seja uma estratégia pedagógica eficiente e de baixo custo no processo de formação de discentes dos cursos de graduação em Enfermagem, da residência multiprofissional e outros cursos de pós-graduação. Dessa forma, pretende-se promover vivências práticas articuladas à teoria no cuidado ao paciente em tratamento hemodialítico, com ênfase na troca de experiências e saberes na construção compartilhada do aprendizado, por meio de um processo avaliativo contínuo, em que sejam consideradas as percepções e sugestões dos discentes, preceptores e tutores.

Neste cenário, Souza e Ferreira (2019) descrevem o preceptor como o profissional do serviço, que aliado ao conhecimento pedagógico, acompanha o desenvolvimento de futuros profissionais da saúde. Os autores defendem que o exercício da prática de preceptoria na área de saúde proporcione um processo de ensino-aprendizagem baseado numa perspectiva teórica e prática acerca do contexto e da realidade em que se realiza. 


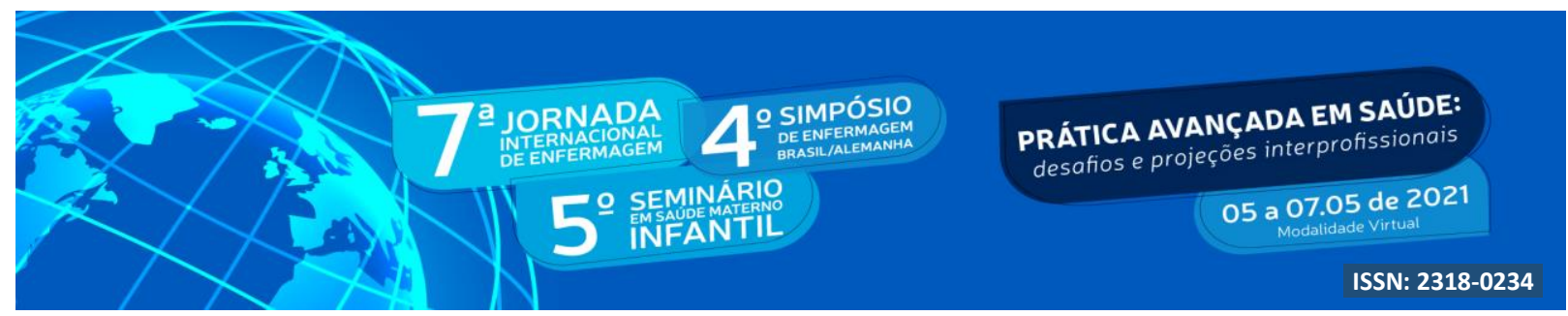

Ressalta-se que ao utilizar as metodologias ativas propostas neste Plano de Preceptoria, todos os envolvidos (preceptores, discentes, tutores e demais profissionais da equipe) poderão refletir e ressignificar as atribuições formativas e o processo de trabalho numa perspectiva ampliada acerca da finalidade e comprometimento com o ensino em saúde no fortalecimento do Sistema Único de Saúde. Acrescenta-se a possibilidade de aprimoramento científico do preceptor a partir da pesquisa e extensão universitária desenvolvida nesses espaços de ensino-aprendizagem, com contribuição social relevante.

Por outro lado, admite-se alguns desafios na implantação do Plano de Preceptoria à medida que as enfermeiras do setor de diálise não foram capacitadas previamente para o exercício da preceptoria, o que requer um planejamento pedagógico permanente para qualificação profissional nesta área, em articulação com os tutores/docentes da UFRN.

Observa-se ainda, a necessidade de adequação das atividades assistenciais e de ensino no setor, com o propósito de favorecer maior disponibilidade de tempo para a prática da preceptoria, minimizando as lacunas entre o cuidar e o ensinar em saúde. Assim como, maior envolvimento da chefia do setor, coordenação dos cursos de graduação e pós-graduação e da gestão do HUOL, a fim de viabilizar pequenas melhorias na infraestrutura do setor para melhor desenvolvimento das atividades de estágio.

O estudo de Lima e Rozendo (2015) também sinalizou para a deficiência de infraestrutura, como a falta de recursos materiais, e estrutura física deficiente, o que dificulta a realização das ações de promoção, controle social e pesquisa.

Na superação desses e de outros desafios encontrados no exercício da preceptoria em saúde, as autoras Souza e Ferreira (2019) destacam que o preceptor ao ampliar seu repertório técnico/profissional e pedagógico, estará favorecendo também a articulação da teoria com a prática, impregnando seu modo de ensinar de novos sentidos, despertando no grupo em que atua um olhar humanizado, sensível e compatível com o cenário em que serão coadjuvantes. Em um panorama geral, a preceptoria engloba dimensões que qualificam o ato formativo, indicando a necessidade de uma formação permanentemente adequada e compatível com a realidade de saúde pública e com as diretrizes curriculares implementadas no atual contexto brasileiro. 


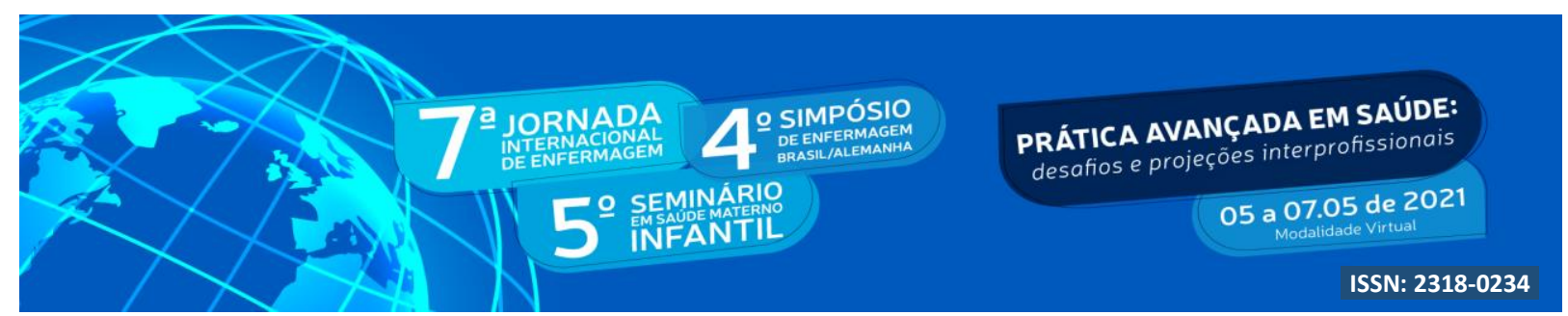

\section{CONCLUSÃO}

Considera-se que o Plano de Preceptoria proposto promoverá o aprimoramento de competências técnico-científicas das preceptoras no desempenho de suas atividades como educadoras, capaz de despertar um novo modo de ensinar, sensível às demandas dos discentes na coprodução do aprendizado. Dessa forma, à medida que o preceptor articula o cuidado em sua essência teórico-prática ao contexto problematizador do estágio, permitirá uma abertura do discente em busca do inédito e do compartilhamento de experiências prévias na constituição do sujeito do conhecimento.

\section{REFERÊNCIAS}

ANTUNES, J.M.; DAHER, D.V.; FERRARI, M.F.M. Preceptoria como lócus de aprendizagem e de coprodução de conhecimento. Rev enferm UFPE on line. [Internet].v.11, n.10, p.3741-3748, 2017. [Citado em 05 Jun 2020]. Disponível em: DOI:

10.5205/reuol.12834-30982-1-SM.1110201706.

BRASIL. Ministério da Educação. Conselho Nacional de Educação. Câmara de Educação Superior. Diretrizes Curriculares Nacionais dos Cursos de Graduação em Enfermagem, Medicina e Nutrição. [Citado em 13 Mar 2020]. Disponível em:

http://portal.mec.gov.br/dmdocuments/ces1133. pdf.

BRASIL. Ministério da Saúde. Conselho Nacional de Saúde. (2016). Resolução no 510, de 7 de abril de 2016. Trata sobre as diretrizes e normas regulamentadoras de pesquisa em ciências humanas e sociais. Diário Oficial da União, Brasília, DF, 24 maio 2016. [Citado em 13 Mar 2020]. Disponível em: http://conselho.saude.gov.br/resolucoes/2016/Reso510.pdf

LIMA, P.A.B.; ROZENDO, C.A. Challenges and opportunities in the Pró-PET-Health preceptorship. Interface (Botucatu).19 Supl 1, p.779-91, 2015. [Citado em 20 Abr 2020]. Disponível em: <http://www.scielo.br/scielo.php?script=sci_arttext\&pid=S1414$32832015000500779 \& \operatorname{lng}=\mathrm{en} \& \mathrm{nrm}=\mathrm{iso}>$.

SOUZA, S.V.; FERREIRA, B.J. Preceptorship: perspectives and challenges in Multiprofessional Residency in Health. ABCS Health Sci. v. 44,n.1,p.15-21, 2019. [Citado em 20 Abr 2020]. Disponível em: https://doi.org/10.7322/abcshs.v44i1.1074

SILVA, Robson Mechel Berto da; MOREIRA, Simone da Nóbrega Tomaz. Estresse e Residência Multiprofissional em Saúde: Compreendendo Significados no Processo de Formação. Rev. bras. educ. med., Brasília, v. 43, n. 4, p. 157-166, Dec. 2019. [Citado em 06 8 Jun 2020]. Disponível em: 


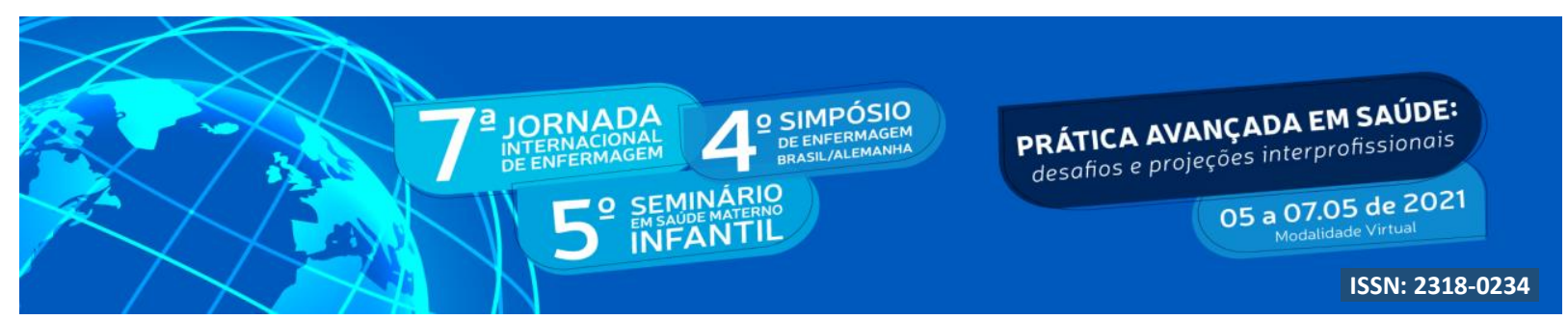

<http://www.scielo.br/scielo.php?script=sci_arttext\&pid=S0100$55022019000400157 \& \operatorname{lng}=$ en\&nrm $=$ iso $>$.

PIUVEZAM, G. Metodologia da Pesquisa. In: Gestão da Política de DST, Aids, Hepatites virais e Tuberculose, Unidade 4. Natal, EDUFRN, 2016. 